\title{
Numerical prediction of the melting curve of $\boldsymbol{n}$-octane
}

\author{
James M. Polson a) and Daan Frenkel \\ FOM Institute for Atomic and Molecular Physics, Kruislaan 407, 1098 SJ Amsterdam, The Netherlands
}

(Received 19 January 1999; accepted 30 April 1999)

\begin{abstract}
We compute the melting curve of $n$-octane using Molecular Dynamics simulations with a realistic all-atom molecular model. Thermodynamic integration methods are used to calculate the free energy of the system in both the crystalline solid and isotropic liquid phases. The Gibbs-Duhem integration procedure is used to calculate the melting curve, starting with an initial point obtained from the free energy calculations. The calculations yield quantitatively accurate results: in the pressure range of 0-100 MPa, the calculated melting curve deviates by only $3 \mathrm{~K}$ from the experimental curve. This deviation falls just within the range of uncertainty of the calculations. (C) 1999 American Institute of Physics. [S0021-9606(99)52128-4]
\end{abstract}

\section{INTRODUCTION}

The calculation of solid-fluid phase coexistence via computer simulation requires special techniques suitable both for high densities and for crystalline solids. The standard approach relies on thermodynamic integration methods to compute the chemical potentials of each phase separately. If the chemical potential is known at a particular state point in either phase, then it can be determined trivially at any other state point using thermodynamic integration along isotherms or isochores, so-called "natural" reversible pathways. A convenient reference point in the fluid phase is in the dilute limit where the system behaves as an ideal gas, and where the free energy can be determined analytically. Alternatively, at sufficiently low density, an appropriate variant of the Widom particle insertion method ${ }^{1,2}$ can be used to calculate the chemical potential. In the solid phase, the standard free energy calculation technique involves thermodynamic integration along an artificial pathway, where the solid is transformed reversibly into an Einstein crystal, in which particles are coupled harmonically to lattice sites; $;^{3}$ the free energy of this reference system can be calculated analytically. This lattice-coupling method is highly versatile and can be applied to arbitrary solids with both continuous and discontinuous potentials. Variants of the method have been applied mainly to systems composed of simple rigid particles, including a variety of hard particle systems such as spheres, 3,4 ellipsoids, ${ }^{5,6}$ spherocylinders, ${ }^{7,8}$ and dimers. ${ }^{9-11}$ Application to any realistic system interacting with a continuous potential is, in principle, straightforward, although the focus until now has been on systems of relatively small, effectively rigid molecules such as $\mathrm{N}_{2},{ }^{12-14} \mathrm{CO}_{2},{ }^{15} \mathrm{~N}_{2} \mathrm{O},{ }^{16}$ and $\mathrm{H}_{2} \mathrm{O} .{ }^{17}$

The $n$-alkane series represents one of the most basic organic series. The development of simulation methods to calculate accurately the equilibrium phase diagram for these systems is of great interest. Recent efforts have focused principally on the simulation of gas-liquid phase equilibrium and critical behavior of alkanes ${ }^{18-21}$ via the combination ${ }^{22,23}$

${ }^{a}$ Current address: Department of Physics, McGill University, Montreal, PQ, H3A 2T8 Canada. of the Gibbs ensemble ${ }^{24-26}$ and configurational biasing ${ }^{27-29}$ Monte Carlo simulation techniques. Clearly, the next important step is an accurate calculation of the solid-liquid coexistence employing the lattice-coupling free energy calculation technique discussed above. In an important step toward this goal, this technique was applied to study the solid-fluid phase equilibrium of simple systems of flexible chain molecules in two recent studies. ${ }^{30,31}$ The first study employed fully flexible chains of hard spheres, ${ }^{30}$ while the second used semi-flexible Lennard-Jones chains. ${ }^{31}$ However, the models employed in both cases only very crudely resemble real chain molecules such as alkanes. A recent study by Malanoski and Monson ${ }^{32}$ focused on the melting behavior of a hard-core model of $n$-alkanes. The simulation data for this hard-core model reference system were subsequently used as input for a mean-field prediction of the $n$-alkane phase diagram. Yet, although the results of such simulations provide insight into the effect of flexibility and steric hindrance on the alkane phase diagram, the simulation results themselves cannot be compared with experimental data. The aim of the present study is to demonstrate the applicability of the method to simulate the melting transition of an alkane system using a realistic all-atom model which is suitable for simulations of crystalline solids and high density liquids, and for which we expect to obtain quantitative agreement with experiment.

The choice of the specific alkane system we study here is governed by two factors. First, we wish to study systems in which a crystalline solid melts to an isotropic fluid directly without passing through any intermediate phases. It is well known that many alkane systems have at least one intermediate "rotator" phase, characterized by rotational disorder about the molecular long axis and gauche defects near the ends of the chain. Rotator phases are present in alkane systems for which the chain length $n$ is given by $n>9$ for odd- $n$ chains and $n>22$ for even- $n$ chains. Second, we note that the determination of the phase equilibrium for a system requires a large number of separate simulations for points along natural pathways (isotherms, isochores) and artificial pathways required for the thermodynamic integration calculations, all of which become computationally very expensive for realis- 
tic all-atom models. Both of these considerations require that we study a relatively small system of short alkane molecules: in the present study we choose $n$-octane.

\section{THEORY AND METHODS}

\section{A. Model}

Simulation studies of alkane systems in the vapor and liquid phases frequently employ united-atom (UA) models, in which the interaction sites consist of composite pseudoatoms composed of a carbon atom plus two (methylene) or three (methyl) hydrogen atoms. The principal advantage of this approach over an all-atom (AA) model, in which every atom is considered to be a separate interaction site, is a considerable reduction in the time to compute the energy and molecular forces. This calculation is the rate-determining step in both Monte Carlo and Molecular Dynamics simulations. However, it has been known for over a decade that UA models are unsuitable for simulations of alkane crystalline solids, where the details of the potential can have a significant effect on the equilibrium properties of the model system. For example, only by employing an AA potential, in which the hydrogen atoms are explicitly represented, is there quantitative agreement between calculated and experimentally measured alkane crystal structures. ${ }^{33,34}$

A simple modification of the standard UA model has been developed by Toxvaerd. ${ }^{35,36}$ In this anisotropic unitedatom (AUA) model, the interaction sites, which are generally placed at the site of the carbon atoms, are displaced slightly in the direction of the hydrogen atoms in the methylene and methyl groups. By tuning the displacement magnitudes, the calculated equation of state for alkane fluids at high densities and pressures, a regime in which predictions using the standard UA models fail drastically, was shown to be in perfect agreement with experiment. Apparently, this straightforward method of accounting for the presence of hydrogen atoms can remove some deficiencies of the UA model. Note that simulations using the AUA model are only marginally more time-consuming than those using an UA model. Thus, this model permits relatively efficient means to simulate accurately a high density fluid. In light of this success, we hoped that the AUA model could be applicable the high density crystalline solid phase, with the same CPU time-saving advantage. Unfortunately, we find that this is not the case. We performed a simple constant-stress simulation of solid $n$-hexane employing the AUA model and observe a crystalline structure which deviates significantly from the experimental structure. The results of the simulation are included in Sec. III. Apparently, the simple remedy of the AUA potential is not sufficient for simulations in the solid phase, and the need to employ an AA model appears unavoidable for solid alkane systems.

Although there are more complicated all-atom potentials, we seek the simplest, empirical potentials which can be efficiently employed in simulations of large systems consisting of many alkanes. These potentials have the following form:

$$
U=U_{\text {bonds }}+U_{\text {angles }}+U_{\text {torsions }}+U_{\text {non-bonded }} \text {. }
$$

Until recently, there have been two different types of models of this class. In the "Ryckaert-McDonald-KleinWilliams" (RMKW) model, ${ }^{34}$ all internal degrees of freedom involving hydrogen atoms, with the exception of methyl group rotations, are frozen. Rotations about $\mathrm{C}-\mathrm{C}$ bonds are governed by cosine power series internal torsional potentials. Intermolecular atoms, and intramolecular atoms separated by three or more carbon atoms interact with an exp-6 potential due to Williams. ${ }^{37}$ This potential has been shown to yield good agreement with experimental alkane crystal structures. By contrast, the "Smith-Karplus" (SK) model ${ }^{38}$ employs a fully flexible representation in which the overall torsional potential is made up of contributions from a single cosine term for each linear set of four bonded atoms in the alkane chain. Atom pairs interact with a Lennard-Jones potential, as well as Coulomb interactions as a result of partial charges placed on the carbon and hydrogen atoms. The latter model, however, performs very poorly in condensed systems, almost certainly a result of the fact that the parameters of this force field were optimized using gas-phase geometries and energetics of small molecules without considering intermolecular interactions.

An alternative model, which is essentially a hybrid of the two described above, is the so-called "Flexible Williams" (FW) potential introduced recently by Tobias et al. ${ }^{39} \mathrm{It}$ employs the Williams exp-6 intermolecular potential of the first model with the fully flexible character of the second. Thus, the molecular geometry and nonbonded parameters are taken from Williams' model IV,${ }^{37}$ and the force constants for bond stretching and angle bending are taken from Smith and Karplus. ${ }^{38}$ Fourier cosine series were fit to the SmithKarplus adiabatic potential for use in the FW model. The advantage of this model is that it removes the cumbersome constraints of the RMKW model, while preserving the Williams potential, which performs well in condensed phases. The FW model been shown to reproduce various experimental data for the solid phase and the high-density liquid phase of alkane systems. ${ }^{39}$ The principal disadvantage is that the inclusion of the bond-stretching and angle-bending terms necessitate, in principle, the use of a smaller time-step in MD simulations. However, this problem can be alleviated with the use of Multiple-Time-Step (MTS) methods. ${ }^{40-42}$ Watanabe and Karplus have shown that, for hydrocarbons, the CPU time of simulations can be decreased by a factor of 3-5 if the reversible reference system propagator algorithm ( $\mathrm{r}-$ RESPA) MTS methods are used. ${ }^{43}$ As we find that the absence of constraints in the FW model somewhat more convenient for performing constant-stress simulations and for the calculation of the free energy of the crystalline solid, we choose to employ the FW model in the present study.

A detailed description of the FW model, as well as the values of all parameters employed (bond lengths and angles, bond-stretching and angle-bending force constants, torsional potential expansion coefficients) can be found in Ref. 39.

\section{B. Free energy calculations}

The alkane melting transition is strongly first-order with a considerable degree of hysteresis in the equation of state. Consequently, it is essential to perform free energy calcula- 
tions in order to determine the melting curve of the system. The conditions for equilibrium coexistence are that the temperatures, pressures and chemical potentials of the coexisting phases be equal. The chemical potential is given by

$$
\mu=\frac{F(\rho)}{N_{\mathrm{ch}}}+\frac{P(\rho)}{\rho},
$$

where $F$ is the Helmoltz free energy of the system, $N_{\text {ch }}$ is the total number of chains and $P(\rho)$ is the pressure as a function of the density of chains $\rho=N_{\mathrm{ch}} / V$. If the Helmoltz free energy at a density $\rho_{0}$ is known, it can be calculated at any other density $\rho$ by the following relation:

$$
\frac{F(\rho)}{N_{\mathrm{ch}}}=\frac{F\left(\rho_{0}\right)}{N_{\mathrm{ch}}}+\int_{\rho_{0}}^{\rho} d \rho^{\prime} \frac{P\left(\rho^{\prime}\right)}{\rho^{\prime 2}} .
$$

Thus, in order to compute the chemical potentials, one must first calculate the absolute Helmoltz free energy at some reference density $\rho_{0}$. The details of this calculation differ for the solid and fluid phases.

\section{Solid phase}

In order to calculate the Helmoltz free energy of a crystalline solid phase, we employ a variant of the method developed by Frenkel and Ladd, ${ }^{3}$ which involves a thermodynamic integration scheme to link a state of a given system along a reversible path to that of another system for which the partition function, and, hence, the Helmholtz free energy, can be calculated analytically. A convenient reference system is the Einstein crystal, where individual noninteracting particles are coupled harmonically to their equilibrium lattice positions

$$
U_{\mathrm{Ein}}=\frac{\alpha}{2} \sum_{i=1}^{N}\left(\vec{R}_{i}-\vec{R}_{i}^{(0)}\right)^{2},
$$

where $\vec{R}_{i}$ is the instantaneous position of the $i$ th particle, and $\vec{R}_{i}^{(0)}$ is the corresponding Einstein crystal lattice position. Further, $N$ is the total number of atoms in the system and is given by $N=n N_{\mathrm{ch}}$, where $n$ is the number of atoms per molecule. Finally, $\alpha$ is the force constant of the Einstein crystal. This quantity is generally set to a value such that the meansquare displacement of the particles in the true crystal and in the reference crystal are approximately the same. This helps to ensure that the numerical implementation of the thermodynamic integration method described below is viable. Further comments on the choice of $\alpha$ for this particular system are given in Sec. III. Note that the Einstein crystal lattice is chosen to have the same structure as that of the true system. Further, note that a "particle" in the context of a system of chains refers to an individual atom on a chain. To carry out the thermodynamic integration, we employ an effective potential:

$$
\widetilde{U}(\lambda)=(1-\lambda) U+\lambda U_{\text {Ein }},
$$

where $U$ is the internal potential energy, and where $\lambda$ is a parameter employed such that $\widetilde{U}(\lambda=0)=U$ and $\widetilde{U}(\lambda=1)$ $=U_{\text {Ein }}$. The free energy difference between the original and reference systems may be calculated by:

$$
\begin{aligned}
F(\lambda=0)-F(\lambda=1) & =-\int_{0}^{1} d \lambda\left(\frac{\partial F(\lambda)}{\partial \lambda}\right) \\
& =-\int_{0}^{1} d \lambda\left\langle\frac{\partial \widetilde{U}}{\partial \lambda}\right\rangle_{\lambda},
\end{aligned}
$$

where the brackets $\langle\ldots\rangle_{\lambda}$ indicate an ensemble average calculated for a particular value of $\lambda$. Thus,

$$
F^{\mathrm{CM}}=F_{\text {Ein }}^{\mathrm{CM}}-\int_{0}^{1} d \lambda\left\langle U_{\mathrm{Ein}}-U\right\rangle_{\lambda}^{\mathrm{CM}} .
$$

The superscript $\mathrm{CM}$ signifies that the center of mass of the crystal is held fixed, a constraint which is required for the thermodynamic integration calculations. Without this constraint, each particle may drift far from its associated Einstein lattice site such that the root-mean-square particle displacement grows to the size of the simulation box in the limit $\lambda \rightarrow 0$. Consequently, the integrand in Eq. (7) becomes sharply peaked close to $\lambda=0$, and the numerical evaluation of the integral would require many simulations at low $\lambda$. Note that bonded atoms are decoupled in the limit of $\lambda=1$, where each atom behaves as a independent harmonic oscillator. To calculate the free energy of the unconstrained crystal, we write

$$
F=\left(F-F^{\mathrm{CM}}\right)+\left(F^{\mathrm{CM}}-F_{\mathrm{Ein}}^{\mathrm{CM}}\right)+F_{\text {Ein }}^{\mathrm{CM}} .
$$

The second term in Eq. (8) is calculated numerically as prescribed in Eq. (7) while the first and third terms can be determined analytically. The complete calculation has been presented elsewhere. ${ }^{44}$ Using this result, it can be shown that the free energy per chain at $\rho_{0}=N_{\mathrm{ch}} / V_{0}$ is given by

$$
\begin{aligned}
\frac{\beta F\left(\rho_{0}\right)}{N_{\mathrm{ch}}}= & 3 n_{\mathrm{C}} \ln \Lambda_{\mathrm{C}}+3 n_{\mathrm{H}} \ln \Lambda_{\mathrm{H}}-\frac{3(N-1)}{2 N_{\mathrm{ch}}} \ln \left(\frac{2 \pi}{\beta \alpha}\right) \\
& -\frac{\ln \left(V_{0} / N_{\mathrm{ch}}\right)}{N_{\mathrm{ch}}}-\frac{\beta}{N_{\mathrm{ch}}} \int_{0}^{1} d \lambda\left\langle U_{\mathrm{Ein}}-U\right\rangle_{\lambda}^{\mathrm{CM}} \\
& +\frac{3}{2 N_{\mathrm{ch}}} \ln \left[N_{\mathrm{ch}} n_{\mathrm{H}} \mu_{\mathrm{H}}^{2}+N_{\mathrm{ch}} n_{\mathrm{C}} \mu_{\mathrm{C}}^{2}\right],
\end{aligned}
$$

where $\beta=1 / k_{B} T$, and $V_{0}=N / \rho_{0}$ is the volume of the system, and $n_{\mathrm{C}}$ and $n_{\mathrm{H}}$ are the number of carbon and hydrogen atoms per molecule. Further, $\Lambda_{i}=h / \sqrt{2 \pi m_{i} k_{B} T}$ are the de Broglie thermal wavelengths for each species, where $m_{i}$ are the atomic masses and $h$ is Plank's constant, and $\mu_{i}=m_{i} / \Sigma_{i} m_{i}$ $=m_{i} /\left(N_{\mathrm{ch}}\left(n_{\mathrm{H}} m_{\mathrm{H}}+n_{\mathrm{C}} m_{\mathrm{C}}\right)\right)$ is the fractional mass of each species.

The chemical potential at an arbitrary density can be computed using Eqs. (2), (3), and (9).

\section{Fluid phase}

The most convenient reference point to calculate the absolute free energy in the fluid phase is in the limit $\rho \rightarrow 0$, where the system behaves as an ideal gas of noninteracting (though still self-interacting) chains. In this limit, the partition function for the system is given by

$$
Z_{\mathrm{IG}}\left(N_{\mathrm{ch}}, V, T\right)=\frac{\left(Z_{\mathrm{ch}}\right)^{N_{\mathrm{ch}}}}{N_{\mathrm{ch}} !}
$$


where $Z_{\mathrm{ch}}$ is the partition function for a single chain. The free energy of the ideal gas is given by:

$$
\begin{aligned}
\frac{\beta F_{\mathrm{IG}}}{N_{\mathrm{ch}}} & =-\ln Z_{\mathrm{ch}}+\ln N_{\mathrm{ch}} ! / N_{\mathrm{ch}} \\
& \approx \beta F_{\mathrm{ch}}+\ln N_{\mathrm{ch}}-1+\frac{\ln \left(2 \pi N_{\mathrm{ch}}\right)}{2 N_{\mathrm{ch}}},
\end{aligned}
$$

where we have employed Stirling's formula plus the firstorder correction, which appears as the last term.

An analytical evaluation of $F_{\mathrm{ch}} \equiv-k_{B} T \ln Z_{\mathrm{ch}}$ for a FW chain is a virtually impossible. Instead we divide the potential into "ideal" and "excess" components, $U_{\mathrm{ch}}^{\mathrm{id}}$ and $U_{\mathrm{ch}}^{\mathrm{ex}}$ $\equiv U^{\mathrm{ch}}-U_{\mathrm{ch}}^{\mathrm{id}}$, respectively, and proceed with the calculation in two steps. First we find a suitable $U_{\mathrm{ch}}^{\text {id }}$ whose free energy $F_{\mathrm{ch}}^{\mathrm{id}}=-k_{B} T \ln Z_{\mathrm{ch}}^{\mathrm{id}}$ can be determined from an analytical calculation of the partition function, $Z_{\mathrm{ch}}^{\mathrm{id}}$, associated with the ideal interactions. In the second step, we employ a numerical method to determine the excess free energy, $F_{\mathrm{ch}}^{\mathrm{ex}} \equiv F_{\mathrm{ch}}$ $-F_{\mathrm{ch}}^{\mathrm{id}}$.

To choose a convenient form for $U_{\mathrm{ch}}^{\mathrm{id}}$, we note first that the difficulty in the evaluation of the complete single chain partition function arises from two sources. The first is the intramolecular nonbonded interactions. In our recent study on the solid-fluid phase equilibria of LJ chain molecules, ${ }^{31}$ we chose $U_{\text {id }}$ to have contributions only from the bonding interactions (bond-stretching and angle-bending), a choice which permits a straightforward analytical calculation of $Z_{\mathrm{ch}}^{\text {id }}$. However, the situation becomes somewhat more complicated in the present case where the presence of hydrogen atoms leads to a branching at the carbon atoms on the chain. The configurational partition function associated with the bonding interactions is difficult to evaluate. Consequently, we define a more convenient form for $U_{\mathrm{ch}}^{\mathrm{id}}$. Specifically, we seek the following properties: (1) the associated partition function $Z_{\mathrm{id}}^{\text {ch }}$ can be calculated in a straightforward manner; (2) specific conformations will have approximately the same ideal energy as the nonbonded energy in the FW model; (3) we require the ability to generate easily molecular configurations from the distribution governed by $U_{\mathrm{ch}}^{\mathrm{id}}$. This last requirement is important for the evaluation of $F_{\mathrm{ex}}^{\mathrm{ch}}$, which we describe below. The definition of $U_{\mathrm{ch}}^{\mathrm{id}}$ and the evaluation of $Z_{\text {id }}^{\text {ch }}$ are described in the Appendix.

The excess single-chain free energy, $F_{\mathrm{ch}}^{\mathrm{ex}} \equiv F_{\mathrm{ch}}-F_{\mathrm{ch}}^{\mathrm{id}}$, is evaluated numerically by averaging the Boltzmann factor of the excess single-chain energy over a series of conformations sampled from a distribution determined by the ideal singlechain energy:

$$
\beta F_{\mathrm{ch}}^{\mathrm{ex}}=-\ln \left\langle\exp \left(-\beta U_{\mathrm{ch}}^{\mathrm{ex}}\right)\right\rangle_{\mathrm{id}} .
$$

We note that

$$
U_{\mathrm{ch}}^{\mathrm{ex}} \equiv U_{\mathrm{ch}}-U_{\mathrm{ch}}^{\mathrm{id}}=\left(U_{\mathrm{ch}}^{\text {bonded }}-U_{\mathrm{ch}}^{\mathrm{id}}\right)+U_{\mathrm{ch}}^{\text {nonbonded }}
$$

and that the choice of $U_{\mathrm{ch}}^{\text {id }}$ and the values of its parameters have been designed to ensure that the difference $U_{\mathrm{ch}}^{\text {bonded }}$ $-U_{\mathrm{ch}}^{\text {id }}$ does not deviate significantly from zero for all conformations generated from the distribution governed by $U_{\mathrm{ch}}^{\mathrm{id}}$. Further, we note that, since the generated conformations are nonself-avoiding, the effect of $U_{\mathrm{ch}}^{\text {nonbonded }}$ on the average can, in principle, cause problems with the statistical accuracy of the evaluation of the average $\left\langle\exp \left(-\beta U_{\mathrm{ch}}^{\mathrm{ex}}\right)\right\rangle_{\mathrm{id}}$ if a large enough majority of the conformations contain at least one overlap of interaction sites. For short chains, this results mainly from the presence of a $\left(\phi_{g_{+}} \phi_{g_{\mp}}\right)$ consecutive pair in the chain, where $\phi_{g_{\alpha}}$ represents a gauche torsional angle of $0^{\circ}<\phi_{g-}<120^{\circ}$ or $240^{\circ}<\phi_{g+}<360^{\circ}$. However, for the calculation for the $n$-octane in the present study, we find a minimum of one such pair in only about $30 \%$ of the generated conformations, and, therefore, this was not a problem. In more problematic calculations of this sort, it may be necessary to employ a Rosenbluth sampling scheme to improve the statistical accuracy of the averaging. ${ }^{29}$

The ideal gas free energy is given by

$$
\begin{aligned}
\frac{\beta F_{\mathrm{IG}}}{N_{\mathrm{ch}}}= & \ln \rho-1+\frac{\ln \left(2 \pi N_{\mathrm{ch}}\right)}{2 N_{\mathrm{ch}}}+3 n_{\mathrm{C}} \ln \Lambda_{\mathrm{C}}+3 n_{\mathrm{H}} \ln \Lambda_{\mathrm{H}} \\
& -\ln Z_{\mathrm{id}, \mathrm{int}}^{\mathrm{ch}}-\ln \left(8 \pi^{2}\right)+\beta F_{\mathrm{ch}}^{\mathrm{ex}},
\end{aligned}
$$

where $Z_{\text {id,int }}^{\text {ch }}$ is defined in the Appendix. Using Eq. (3), and the fact that $P_{\mathrm{IG}}=N_{\mathrm{ch}} k_{B} T / V$, it is trivial to show that

$$
\frac{\beta F(\rho)}{N_{\mathrm{ch}}}=\frac{\beta F_{\mathrm{IG}}(\rho)}{N_{\mathrm{ch}}}+\int_{0}^{\rho} d \rho^{\prime}\left[\frac{\beta P\left(\rho^{\prime}\right)-\rho^{\prime}}{\left(\rho^{\prime}\right)^{2}}\right] .
$$

One further relation that is employed in the present study is that for the free energy difference evaluated along an isochore between systems at temperatures $T_{1}$ and $T_{2}$ and constant density $\rho$ :

$$
\frac{F_{1}}{T_{1}}-\frac{F_{2}}{T_{2}}=\int_{1 / T_{2}}^{1 / T_{1}} d(1 / T) E(\rho, T),
$$

where $E(\rho, T)$ is the total energy of the system.

Finally, the chemical potential for the fluid phase can be calculated using Eqs. (2), (14), (15) and (16).

\section{Calculation of the phase boundaries}

Using the expressions derived in the previous section, it is possible to calculate $\mu(P)$ for fixed $T$ for each phase. The intersection of these functions determines the location where the conditions for thermodynamic coexistence are satisfied and gives a single point on the melting curve. Since the free energy calculations required to obtain this single point are computationally very expensive, we do not repeat them in order to obtain a full line of points. Instead, we employ the Gibbs-Duhem integration scheme developed by Kofke $e^{45,46}$ to trace out the phase boundaries, without the need to compute further free energies. The simplest variant of this method involves the integration of the standard ClaussiusClapeyron equation,

$$
\left(\frac{d P}{d \beta}\right)_{\text {coexist }}=-\frac{\Delta h}{\beta \Delta v},
$$

where $\Delta v=v_{2}-v_{1}$ and $\Delta h=h_{2}-h_{1}$ are the differences in molar volume and enthalpy between the two phases, respectively. As the Claussius-Clapeyron equation is a first-order differential equation, it can be integrated to compute the coexistence curve provided one point on the curve is known. 


\section{Simulation details}

The simulations employed systems of $N_{\mathrm{ch}}=64$ octane molecules in the fluid phase, and $N_{\mathrm{ch}}=50$ chains in the solid phase. The use of a small system size is an unfortunate necessity when employing an AA alkane model for which a large number of separate simulations must be carried out. Clearly, there will be some finite-size effects present. However, considering the large number of degrees of freedom of this system, the effects should be much smaller than for those of atomic systems of the same size, and should presumably have only a slight effect of the coexistence points. Since an explicit calculation of the finite-size scaling of the melting curve results is prohibitively time-consuming, it is not possible to give a quantitative estimate of the finite-size effects.

The 64 octane molecules in the fluid phase were placed in a cubic box. The equilibration procedure depended on the density and temperature. Simulations were carried out on the supercritical isotherm at $T=650 \mathrm{~K}$ for a wide density range. At low densities $\left(\rho<0.40 \mathrm{~g} / \mathrm{cm}^{3}\right)$, the octane chains were initially centered on points of a $4 \times 4 \times 4$ bcc lattice in their all-trans extended conformation. NVT simulations with these systems were carried out for both equilibration and production. At higher densities, a final configuration of a lower density run was used as an initial configuration, and the system was equilibrated by compression through an NPT simulation to a desired density or pressure. After equilibration, an NVT production simulation was carried out. At lower temperatures, a problem arises due to the very slow relaxation toward conformational equilibrium when the trans-gauche energy barrier becomes large enough compared to $k_{B} T$. To circumvent this problem, we employed a method similar to that used by Tobias et al. ${ }^{39}$ in which starting configurations were obtained by isothermally compressing randomly chosen vacuum chain conformers (i.e., chains in conformations sampled at different times in a single-chain MD simulation) initially placed on a cubic lattice. As the conformational behavior of flexible molecules is generally found to be only marginally affected by condensation ${ }^{47}$ this will likely accelerate the equilibrium process. In the study by Tobias et $a l .{ }^{39}$ the conformers were obtained from sampling from a one-molecule MD simulation. In the present study, we chose instead to conduct single-chain configurational bias MC simulations using a UA model and then to use sampled configurations to initiate the MD simulations for one point on the $T=230 \mathrm{~K}$ isotherm at $P=50 \mathrm{MPa}$. The final configuration from this simulation was used as an initial configuration for the next higher and lower pressure, and so on, along the isotherm. NPT simulations were conducted at state points along the $T=230 \mathrm{~K}$ isotherm and along the phase boundary in the Gibbs-Duhem integration, while NVT simulations were performed along the $\rho=0.81 \mathrm{~g} / \mathrm{cm}^{3}$ isochore between $T=230$ and $650 \mathrm{~K}$. All liquid phase simulations employed equilibration times of at least $100 \mathrm{ps}$ and production runs of at least $50 \mathrm{ps}$.

For the solid phase, the simulation cell contained $50 \mathrm{oc}-$ tane chains arranged in two layers of $5 \times 5$ molecules. Constant-stress NPT simulations were carried out at points along the $T=230 \mathrm{~K}$ isotherm and along the phase boundary in the Gibbs-Duhem integration. The initial configuration for one octane crystal simulation was taken from the previously published x-ray crystal structure. ${ }^{48}$ Initially configurations for other simulations obtained from the final configuration of the previous simulation along the isotherm or phase boundary. All solid phase simulations used equilibration times of at least $25 \mathrm{ps}$, and production runs of at least $50 \mathrm{ps}$.

Because we choose a fully flexible model to describe the alkane system, it is essential to employ a Multiple-Time-Step (MTS) method ${ }^{40-42}$ to integrate the equations of motion in order to avoid the need to use a very small time-step. We choose the reference force to consist of the $\mathrm{CH}$ and $\mathrm{CC}$ bond-stretching forces, and the $\mathrm{CCC}, \mathrm{CCH}$ and $\mathrm{HCH}$ anglebending forces. We employ the integrators developed by Martyna et al. ${ }^{42}$ for all NVT, NPT and constant-stress NPT simulations. The fictitious masses for the thermostat and barometer variables were chosen according to the prescription by Martyna et al. ${ }^{49}$ with a thermostat time scale of $0.62 \mathrm{ps}$ and a barostat time scale of 1.26 ps. A large time-step of $\Delta t=1$ fs subdivided into $n_{s}=4$ small time-steps of $\delta t$ $=\Delta t / n_{s}=0.25 \mathrm{fs}$ was employed.

Standard periodic boundary conditions were employed. The exp-6 long-range interactions were truncated at $10 \AA$, and long-range corrections were included in the calculation of the energies and pressures to compensate for this truncation.

To calculate the free energy of the crystalline solid, we evaluate the integral appearing in Eq. (7) using the ten-point Gauss-Legendre quadrature method. First the average cell shape and atom positions were calculated to provide the reference lattice used in the calculations. Then, the average energy difference $\left\langle U_{\text {Ein }}-U\right\rangle_{\lambda}^{\mathrm{CM}}$ was evaluated at the appropriate ten values of $\lambda$ at constant volume. We note that when $\lambda \neq 0$, there is an external force acting on the system due to the coupling between the atoms and their lattice positions. Consequently, the center of mass is not fixed. To constrain the center of mass in these calculations we essentially employ the Shake ${ }^{50}$ and Rattle ${ }^{51}$ algorithm, which in practice, simply amounts to subtracting the difference between the current and initial center of mass position from all particle positions, and the current center of mass momentum from all particle momenta, in a single iteration at the appropriate place in the equation of motion integration algorithm.

We perform the Gibbs-Duhem integration with respect to $\beta$, i.e., integration of Eq. (17), using a second-order predictor corrector method, and with an integration step of $d \beta$ $=0.0001\left(\mathrm{Kk}_{B}\right)^{-1}$, which corresponds to an increment of approximately $5 \mathrm{~K}$ in the temperature range considered here.

\section{RESULTS}

An important test of the suitability of a particular molecular model to calculate accurately the free energy of a crystalline solid is that the crystal structure measured in the simulation closely matches that measured experimentally. As discussed in Sec. II A, standard UA models are unable to predict correctly the measured crystal structure. Further, although Toxvaerd's AUA model improves the results for the calculation of the equation of state for fluids at high pres- 
TABLE I. Unit cell parameters for solid $n$-hexane at $T=158 \mathrm{~K}$ obtained by experiment and simulation $(P=0.1 \mathrm{MPa})$ with the Toxvaerd AnisotropicUnited-Atom (AUA) model.

\begin{tabular}{lcc}
\hline \hline & Expt. $^{\mathrm{a}}$ & MD \\
\hline$\rho\left(\mathrm{g} / \mathrm{cm}^{3}\right)$ & 0.888 & 0.891 \\
$a(\AA)$ & 4.17 & 3.93 \\
$b(\AA)$ & 4.7 & 5.56 \\
$c(\AA)$ & 8.57 & 8.19 \\
$\alpha\left({ }^{\circ}\right)$ & 96.6 & 74.0 \\
$\beta\left({ }^{\circ}\right)$ & 87.2 & 103.0 \\
$\gamma\left({ }^{\circ}\right)$ & 103.0 & 110.0 \\
\hline \hline
\end{tabular}

${ }^{\mathrm{a}}$ Reference 55 .

sures and densities, it also suffers from the same problem for the solid. In Table I, we present the results from a constantstress NPT simulation for a system of solid $n$-hexane at $P$ $=0.1 \mathrm{MPa}$. The deviation of the unit cell parameter values from the corresponding experimentally measured values clearly illustrates the unsuitability of using this model for solid-phase simulations. By contrast, the results of another constant-stress NPT simulation of solid $n$-octane using the FW model, presented in Table II, are considerably more favorable, and demonstrate the need to employ an AA model.

Simulation snapshots of $n$-octane in the fluid and crystalline solid phases are shown in Figs. 1 and 2, respectively.

The evaluation of the free energy of the system at state points in the liquid and solid phases requires several separate calculations for each phase. Below, we give the results of each separate calculation. The values of the various quantities are also listed in Table III for convenience.

Figure 3 shows the calculated points on the isotherms used for thermodynamic integration calculations in this study. We seek the free energy as a function of density $F(\rho)$ in the solid and fluid phases at $T_{1}=230 \mathrm{~K}$, somewhat larger than the $n$-octane experimental melting temperature of 216 $\mathrm{K}$. To this end, we have calculated isotherms at this temperature for both phases. The free energy of the solid is calculated at one point on the isotherm using the Einstein crystal thermodynamic integration method described in Sec. II B 1. The fluid phase free energy must be calculated by thermodynamic integration along an isotherm to the dilute limit of $\rho \rightarrow 0$. To avoid the problem of passing through the gasliquid two-phase region, we have chosen to integrate along a

TABLE II. Unit cell parameters for solid $n$-octane obtained by experiment and simulation.

\begin{tabular}{lccc}
\hline \hline & Expt. $^{\mathrm{a}}$ & $\mathrm{MD}^{\mathrm{b}}$ & $\mathrm{MD}^{\mathrm{c}}$ \\
\hline$T(\mathrm{~K})$ & 190.0 & 190.0 & 180.0 \\
$\rho\left(\mathrm{g} / \mathrm{cm}^{3}\right)$ & 0.891 & 0.915 & 0.916 \\
$a(\AA)$ & 4.22 & 4.20 & 4.23 \\
$b(\AA)$ & 4.79 & 4.62 & 4.58 \\
$c(\AA)$ & 11.02 & 11.07 & 11.08 \\
$\alpha\left({ }^{\circ}\right)$ & 94.7 & 95.0 & 95.0 \\
$\beta\left({ }^{\circ}\right)$ & 84.3 & 84.6 & 84.7 \\
$\gamma\left({ }^{\circ}\right)$ & 105.8 & 104.1 & 104.2 \\
\hline \hline
\end{tabular}

${ }^{\mathrm{a}}$ Reference 48.

${ }^{b}$ Present study. $P=0.1 \mathrm{MPa}$.

${ }^{\mathrm{c}}$ Reference 39. $P=0$.

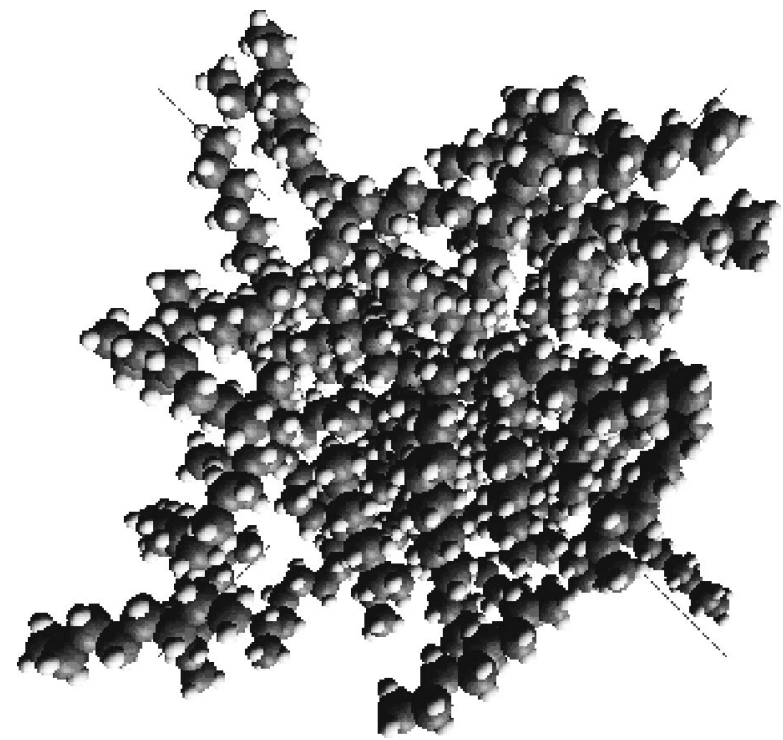

FIG. 1. Simulation snapshot of a system of $N_{\mathrm{ch}}=64 n$-octane chains in the isotropic liquid phase at $\rho=0.793 \mathrm{~g} / \mathrm{cm}^{3}$ and $T=230 \mathrm{~K}$.

supercritical isotherm at $T_{2}=650 \mathrm{~K}$, significantly above the critical point temperature of $T_{c}=568.6 \mathrm{~K}$. To connect the $T_{1}=230 \mathrm{~K}$ isotherm to the $T_{2}=650 \mathrm{~K}$ isotherm, we measure the energy at points along an isochore at $\rho_{a}=0.81 \mathrm{~g} / \mathrm{cm}^{3}$ and employ Eq. (16) to calculate the free energy difference.

The first step is the evaluation of the single-chain excess free energy using the method described in Sec. II B 2. We employ a Monte Carlo procedure to generate a series of configurations from a distribution corresponding to the $U_{\mathrm{ch}}^{\text {id }}$ and compute the average in Eq. (12). Using 10 blocks of 200000 chain configurations per block, we determine that $\beta_{2} F_{\text {ch }}^{\mathrm{ex}}$ $=-0.688(1)$, listed as item (1) in Table III for the fluid, where $\beta_{2}=1 / k_{B} T_{2}$.

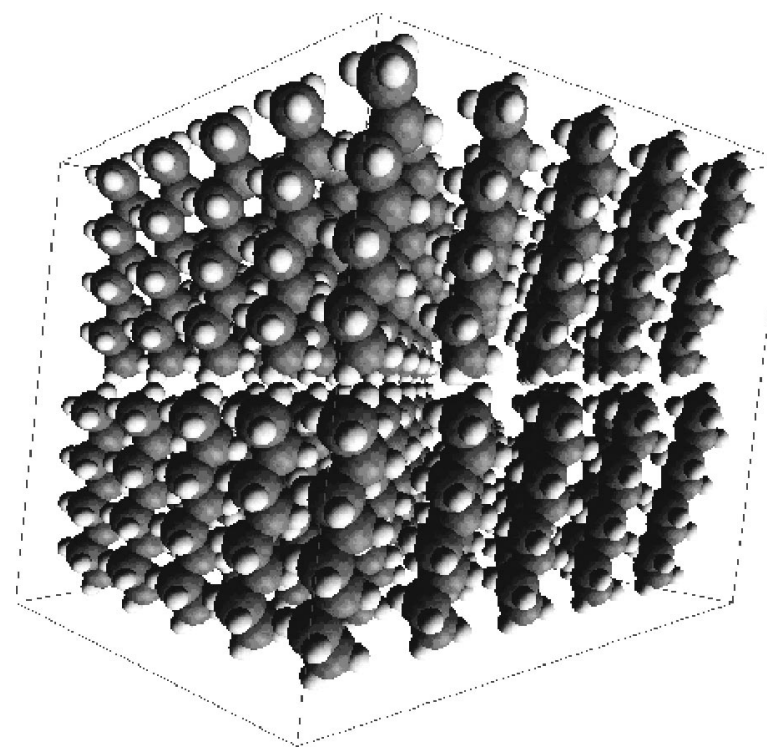

FIG. 2. Simulation snapshot of a system of $N_{\mathrm{ch}}=50 n$-octane chains in the crystalline solid phase at $\rho=0.906 \mathrm{~g} / \mathrm{cm}^{3}$ and $T=230 \mathrm{~K}$. The chains are arranged in two layers of $5 \times 5$ chains per layer. 
TABLE III. Calculated quantities relevant for free energy calculations. $\beta_{i}$ $=1 /\left(k_{B} T_{i}\right)$, where $T_{1}=230 \mathrm{~K}$ and $T_{2}=650 \mathrm{~K} . \Lambda_{i}^{\alpha} \equiv h /\left(2 \pi m_{\alpha} k_{B} T_{i}\right)^{1 / 2}$ is the de Broglie thermal wavelength for $\alpha=\mathrm{C}$ and $\mathrm{H}$ at $T_{1}=230 \mathrm{~K}$ and $T_{2}$ $=650 \mathrm{~K} . N_{\mathrm{ch}}$ is the number of alkane chains in the system, and $n_{\mathrm{C}}$ and $n_{\mathrm{H}}$ are the number of carbon atoms and hydrogen atoms, respectively, per chain. $P(\rho)$ is the pressure as a function of density of chains $N_{\mathrm{ch}} / V . F_{\mathrm{ch}}^{\mathrm{ex}}$ is the excess single chain free energy defined in Eq. (12). $F_{\mathrm{IG}}(\rho)$ is the ideal gas free energy at density $\rho$ and is defined in Eq. (14). $\left\langle U_{\mathrm{Ein}}-U\right\rangle_{\lambda}^{\mathrm{CM}}$ is the average difference between the energy of a crystal, $U$, and an Einstein crystal with the same equilibrium lattice, $U_{\text {Ein }}$ [see Eq. (4)], calculated using an effective potential given by Eq. (5) for $\lambda \in[0,1]$, and subject to the constraint of a fixed center of mass. For the solid phase, $F$ is the free energy of the unconstrained crystal, $F^{\mathrm{CM}}$ is the free energy of the crystal with a constrained center of mass, and $F_{\text {Ein }}^{\mathrm{CM}}$ is the free energy of an Einstein crystal with a constrained center of mass. Finally, $\rho_{a}=0.810 \mathrm{~g} / \mathrm{cm}^{3}$, and $\rho_{b}$ $=0.904 \mathrm{~g} / \mathrm{cm}^{3}$.

\begin{tabular}{rlc}
\hline \hline \multicolumn{3}{c}{ Fluid } \\
\hline$(1)$ & $\beta_{2} F_{\mathrm{ch}}^{\mathrm{ex}} / N_{\mathrm{ch}}$ & $-0.688(1)$ \\
$(2)$ & $\beta_{2} F_{\mathrm{IG}}\left(\rho_{a}\right) / N_{\mathrm{ch}}-3 n_{\mathrm{C}} \ln \Lambda_{2}^{\mathrm{C}}-3 n_{\mathrm{H}} \ln \Lambda_{2}^{\mathrm{H}}$ & $75.995(1)$ \\
$(3)$ & $\int_{0}^{\rho_{a}} d \rho^{\prime}\left[\beta P\left(\rho^{\prime}\right)-\rho^{\prime}\right] /\left(\rho^{\prime}\right)^{2}$ & $1.1(1)$ \\
$(4)$ & $\beta_{2} F\left(\rho_{a}\right) / N_{\mathrm{ch}}-3 n_{\mathrm{C}} \ln \Lambda_{2}^{\mathrm{C}}-3 n_{\mathrm{H}} \ln \Lambda_{2}^{\mathrm{H}}$ & $77.1(1)$ \\
$(5)$ & $\int_{1 / T_{2}}^{1 / T_{1}} d(1 / T) E\left(\rho_{a}, T\right) /\left(N_{\mathrm{ch}} k_{B}\right)$ \\
$(6)$ & $\beta_{1} F\left(\rho_{a}\right) / N_{\mathrm{ch}}-3 n_{\mathrm{C}} \ln \Lambda_{2}^{\mathrm{C}}-3 n_{\mathrm{H}} \ln \Lambda_{2}^{\mathrm{H}}$ & $64.97(3)$ \\
$(7)$ & $3 n_{\mathrm{C}} \ln \left(\Lambda_{2}^{\mathrm{C}} / \Lambda_{1}^{\mathrm{C}}\right)+3 n_{\mathrm{H}} \ln \left(\Lambda_{2}^{\mathrm{H}} / \Lambda_{1}^{\mathrm{H}}\right)$ & $142.1(1)$ \\
$(8)$ & $\beta_{1} F\left(\rho_{a}\right) / N_{\mathrm{ch}}-3 n_{\mathrm{C}} \ln \Lambda_{1}^{\mathrm{C}}-3 n_{\mathrm{H}} \ln \Lambda_{1}^{\mathrm{H}}$ & -40.517 \\
& \multicolumn{1}{c}{$\operatorname{Solid}$} \\
& \multicolumn{1}{c}{$101.5(1)$} \\
$(9)$ & $\left(\beta_{1} / N_{\mathrm{ch}}\right) \int_{0}^{1} d \lambda\left\langle U_{\mathrm{Ein}}-U\right\rangle_{\lambda}^{\mathrm{CM}}$ \\
$(10)$ & $\left(\beta_{1} / N_{\mathrm{ch}}\right)\left[\left(F\left(\rho_{b}\right)-F^{\mathrm{CM}}\left(\rho_{b}\right)\right)+F_{\mathrm{Ein}}^{\mathrm{CM}}\right]$ & $13.1(1)$ \\
& $-3 n_{\mathrm{C}} \ln \Lambda_{1}^{\mathrm{C}}-3 n_{\mathrm{H}} \ln \Lambda_{1}^{\mathrm{H}}$ & 114.957 \\
$(11)$ & $\beta_{1} F\left(\rho_{b}\right) / N_{\mathrm{ch}}-3 n_{\mathrm{C}} \ln \Lambda_{1}^{\mathrm{C}}-3 n_{\mathrm{H}} \ln \Lambda_{1}^{\mathrm{H}}$ \\
\hline \hline
\end{tabular}

Using Eq. (14), we determine the ideal gas free energy at $T_{2}=650 \mathrm{~K}$ at a density of $\rho_{a}=0.81 \mathrm{~g} / \mathrm{cm}^{3}$. We calculate $Z_{\mathrm{ch}, \text { int }}^{\text {id }}$ through a numerical calculation of the integrals in Eq. (A8). We find that $\beta_{2} F_{\mathrm{IG}} / N_{\mathrm{ch}}-3 n_{\mathrm{C}} \ln \Lambda_{2}^{\mathrm{C}}-3 n_{\mathrm{H}} \ln \Lambda_{2}^{\mathrm{H}}$ $=75.995$ [item (2) in Table III], where $\Lambda_{i}^{\alpha}$ is the de Broglie thermal wavelength for a particle of type $\alpha=\mathrm{C}, \mathrm{H}$ at temperature $T_{i}$. These kinetic terms involving the thermal wavelengths which arise from the integration of the momenta in the partition function play no essential role in the free energy calculation. However, because the calculations were carried

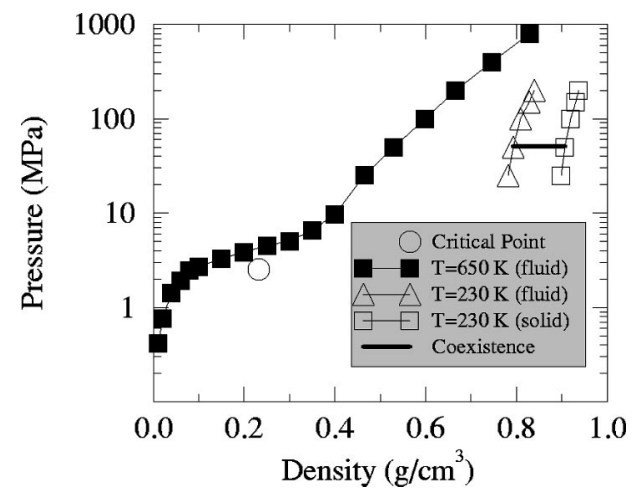

FIG. 3. Calculated isotherm for $n$-octane at $T=650 \mathrm{~K}$ for the fluid phase, and at $T=230 \mathrm{~K}$ for solid and liquid phases. The gas-liquid critical point at $T=568.4 \mathrm{~K}$ is also labeled, as well as the solid-liquid coexistence tie line at $P=50.82 \mathrm{MPa}$ for $T=230 \mathrm{~K}$.

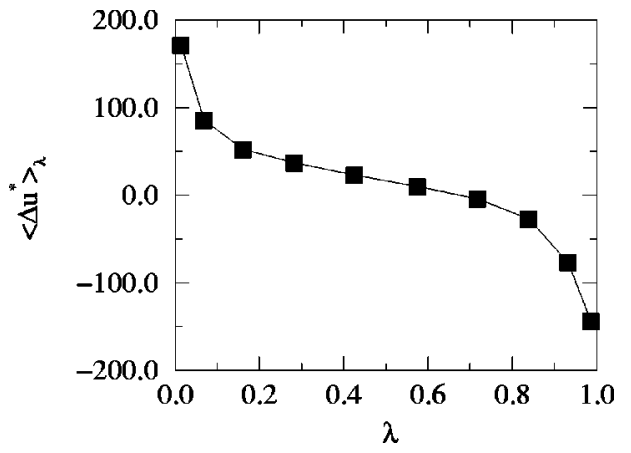

FIG. 4. Calculated $\left\langle\Delta u^{*}\right\rangle_{\lambda} \equiv\left(\beta / N_{\mathrm{ch}}\right)\left\langle U_{\text {Ein }}-U\right\rangle_{\lambda}^{\mathrm{CM}}$ used in the ten-point Gauss-Legendre integration of Eq. (7) at $\rho=0.904 \mathrm{~g} / \mathrm{cm}^{3}$ and $T=230 \mathrm{~K}$. An Einstein crystal force constant of $\alpha=30000 \mathrm{~K} / \AA^{2}$ was used.

out at two different temperatures, we include them explicitly to avoid confusion.

To determine the free energy of the true fluid at $\rho_{a}$ $=0.81 \mathrm{~g} / \mathrm{cm}^{3}$, we employ Eq. (15). Using a eighth-order polynomial to fit the $T_{2}=650 \mathrm{~K}$ isotherm, we find that the integral in Eq. (15) has a value of 1.1(1) [item (3) in Table III]. Consequently, the free energy of the fluid has a value of $\beta_{2} F\left(\rho_{a}\right) / N_{\mathrm{ch}}=77.1(1)$ [item (4) in Table III]. To connect this pathway with the $230 \mathrm{~K}$ isotherm, we employ Eq. (16) and carry out the integration along an isochore at $0.81 \mathrm{~g} / \mathrm{cm}^{3}$. We employ a five point Gauss-Legendre integration scheme to evaluate the integral and find that $\left(\beta_{1} F\left(T_{1}\right)\right.$ $\left.-\beta_{2} F\left(T_{2}\right)\right) / N_{\mathrm{ch}}=64.9(1)$ at $\rho=0.81 \mathrm{~g} / \mathrm{cm}^{3}$. Consequently, the free energy of the fluid less the kinetic factors in Eq. (14), $3 n_{\mathrm{C}} \ln \Lambda_{2}^{\mathrm{C}}+3 n_{\mathrm{H}} \ln \Lambda_{2}^{\mathrm{H}}$, has value 142.1(1) [item (6) in Table III]. To provide a more convenient reference point to compare with the solid phase, we evaluate the difference between the free energy and the kinetic terms, where the latter are evaluated at $T_{1}=230 \mathrm{~K}$. Consequently, we add a value of $3 n_{\mathrm{C}} \ln \left(\Lambda_{2}^{\mathrm{C}} / \Lambda_{1}^{\mathrm{C}}\right)+3 n_{\mathrm{H}} \ln \left(\Lambda_{2}^{\mathrm{H}} / \Lambda_{1}^{\mathrm{H}}\right)=-40.517 \quad$ [item $\quad$ (7) in Table III], and find that the free energy of the liquid at $\rho_{a}$ $=0.81 \mathrm{~g} / \mathrm{cm}^{3}$ and $T_{1}=230 \mathrm{~K}$ has a value $\beta_{1} F\left(\rho_{a}\right) / N_{\mathrm{ch}}$ $-3 n_{\mathrm{C}} \ln \Lambda_{1}^{\mathrm{C}}-3 n_{\mathrm{H}} \ln \Lambda_{1}^{\mathrm{H}}=101.5$ (1) [item (8) in Table III].

Figure 4 shows the calculated $\left\langle\Delta u^{*}\right\rangle_{\lambda} \equiv\left(\beta / N_{\mathrm{ch}}\right)\left\langle U_{\text {Ein }}\right.$ $-U\rangle_{\lambda}^{\mathrm{CM}}$ used in the ten-point Gauss-Legendre evaluation of the integral in Eq. (7). We note that for the value of Einstein crystal force constant that was used $\left(\alpha=30000 \mathrm{~K} / \AA^{2}\right)$, the function $\left\langle\Delta u^{*}\right\rangle_{\lambda}$ has a positive peak near $\lambda=0$, and a small negative peak near $\lambda=1$. This behavior was also noted in our previous study of semi-flexible Lennard-Jones chains in the case of stiff bonds. ${ }^{31}$ If $\alpha$ is small, then, in the limit where $\lambda \rightarrow 1$ and $\widetilde{U}=U_{\text {Ein }}$, deviations in bond lengths and bond angles from their equilibrium values will be significant, and lead to a large value of $U$ and, therefore, a possibly sharp reduction in $\left\langle\Delta u^{*}\right\rangle_{\lambda}$. If $\alpha$ is too large, then, in the limit where $\lambda \rightarrow 0$ and $\widetilde{U}=U$, the individual atoms may deviate significantly from their equilibrium lattice positions, leading to a large value of $U_{\text {Ein }}$ and, therefore, a high value of $\left\langle\Delta u^{*}\right\rangle_{\lambda}$. These two cases lead to peaks at $\lambda=0$ and 1 . As in the case of the Lennard-Jones chain system with stiff bonds, ${ }^{31}$ we find that there is no intermediate value of $\alpha$ that simultaneously removes both peaks and yields a smoothly varying $\left\langle\Delta u^{*}\right\rangle_{\lambda}$ over the full range of $\lambda$. Nevertheless, the 


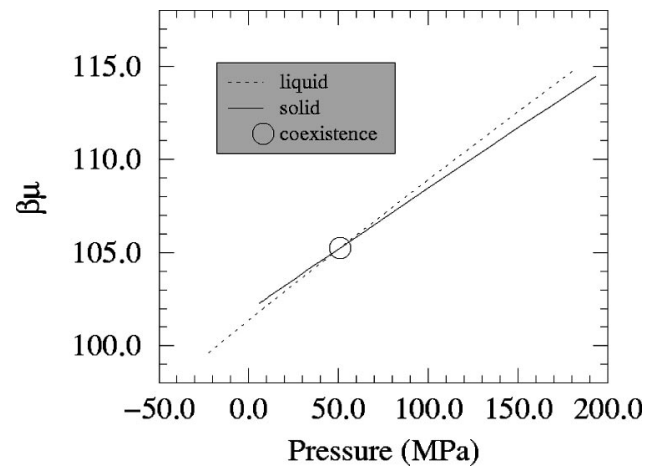

FIG. 5. Solid and fluid branches of the reduced chemical potential $\beta \mu$ as a function of the pressure $P$ at $T=230 \mathrm{~K}$.

value of $\alpha=30000 \mathrm{~K} / \AA^{2}$ was found to minimize each of the two peaks. It is possible that the presence of the peaks could present problems for the ten-point Gauss-Legendre numerical integration. Since the calculation of the ten points in Fig. 4 was extremely time-consuming, we did not repeat the numerical integration for more points, nor did we attempt to determine a suitable transformation to a different integration variable such that the integrand is a more smoothly varying function. However, we note that when such approaches were tested in our previous study ${ }^{31}$ for the stiff-bond LennardJones chain solid, for which $\left\langle\Delta u^{*}\right\rangle_{\lambda}$ also displayed the same features, the 10-point integration was found to be sufficiently accurate.

The integral has a value of $\int_{0}^{1} d \lambda\left\langle\Delta u^{*}\right\rangle_{\lambda}^{\mathrm{CM}}=13.1(1)$ [item (9) in Table III]. We subtract this from the quantity $\left(\beta_{1} / N_{\mathrm{ch}}\right)\left[\left(F\left(\rho_{b}\right)-F^{\mathrm{CM}}\left(\rho_{b}\right)\right)+F_{\mathrm{Ein}}^{\mathrm{CM}}\right]$, which is defined by Eqs. (8) and (9), and which has a value of 114.957 [item (10) in Table III]. Thus, the free energy of the crystalline solid at $\rho_{b}=0.904 \mathrm{~g} / \mathrm{cm}^{3}$ and $T_{1}=230 \mathrm{~K}$ is given by $\beta_{1} F\left(\rho_{b}\right) / N_{\mathrm{ch}}$ $-3 n_{\mathrm{C}} \ln \Lambda_{1}^{\mathrm{C}}-3 n_{\mathrm{H}} \ln \Lambda_{1}^{\mathrm{H}}=101.9$ (1) [item (11) in Table III].

From the calculations described above, the absolute free energy of the $n$-octane system is known for one point on each of the solid and fluid branches of the $230 \mathrm{~K}$ isotherm. As we have calculated several points along the isotherm, we can determine the chemical potential of each phase as a function of density or pressure at this temperature using Eqs. (2) and (3). Solid and liquid branches of $\mu(P)$ are shown in Fig. 5. The intersection point at $P=50.8 \mathrm{MPa}$ gives the pressure at coexistence between the two phases at $230 \mathrm{~K}$. This corresponds to densities of $\rho_{l}=0.790 \mathrm{~g} / \mathrm{cm}^{3}$ for the liquid phase and $\rho_{s}=0.910 \mathrm{~g} / \mathrm{cm}^{3}$ for the solid phase.

With one point on the coexistence boundary, we obtain other points along the curve via Gibbs-Duhem integration, i.e., by integrating Eq. (17). The results are tabulated in Table IV. The melting curve in the $P-T$ plane is shown in Fig. 6. The curve is almost perfectly linear, which is simply a reflection of the narrow $20 \mathrm{~K}$ temperature range considered. To estimate the uncertainties in the calculated melting curve data, we note from Fig. 5 that small relative displacements of the $\mu(P)$ curves will shift the position of the intersection of the curves over a wide range in pressure. Consequently, we assume that the principal source of error is the uncertainty in the calculation of the absolute free energy at the reference points on the liquid and solid branches of the
TABLE IV. Calculated liquid-solid coexistence points for $n$-octane.

\begin{tabular}{crcc}
\hline \hline$T(\mathrm{~K})$ & $P(\mathrm{MPa})$ & $\rho_{f}\left(\mathrm{~g} / \mathrm{cm}^{3}\right)$ & $\rho_{s}\left(\mathrm{~g} / \mathrm{cm}^{3}\right)$ \\
\hline 219.89 & 2.4 & 0.775 & 0.911 \\
224.83 & 25.5 & 0.781 & 0.909 \\
230.00 & 50.8 & 0.790 & 0.910 \\
235.41 & 77.9 & 0.803 & 0.916 \\
241.09 & 104.8 & 0.806 & 0.919 \\
\hline \hline
\end{tabular}

$230 \mathrm{~K}$ isotherm prior to thermodynamic integration along the isotherm branches. The uncertainty of the free energy for both the solid and liquid reference points is $\delta F=0.1 k_{B} T_{1}$ per chain. Applying a relative shift of the chemical potential of $2 \delta F$ shifts the location of the intersection of the $\mu(P)$ curves in Fig. 5 by approximately $\delta P=20 \mathrm{MPa}$. Next, we conduct simulations for the solid and liquid phases at 50.8 $\mathrm{MPa} \pm \delta P$ to measure the enthalpy and molar volume differences between the phases and take a linear approximation of Eq. (17) to construct shifted melting curves. The region between the two calculated gives a reasonably accurate measure of the uncertainty of the position of the melting curve; this region is colored gray in the figure.

Superimposed on the phase diagram are three points on the melting curve taken from the experimental differential thermal analysis study of $n$-octane of Würflinger. ${ }^{52}$ While the pressure dependence of the melting temperature in that study was measured to pressures of up to $300 \mathrm{MPa}$, Fig. 6 includes only those data which fall within the $0-100 \mathrm{MPa}$ pressure range considered in the calculations. The experimental "curve" is essentially parallel to the calculated curve, and shifted to lower temperatures by approximately $3 \mathrm{~K}$. Further, we note that the experimental data points lie within the shaded area for the temperature range considered here.

Given that the temperature scale in Fig. 6 is highly expanded, the quantitative agreement between the experimental and calculated melting curves is striking: the melting temperatures have been calculated to within $2 \%$ of their true values in the pressure range considered. As stated above, the

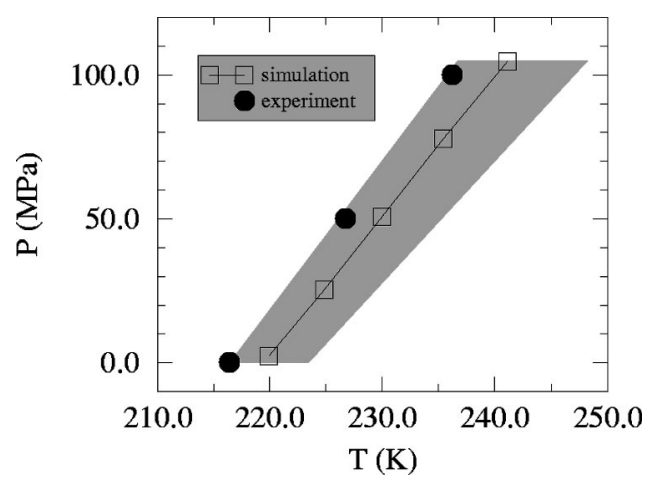

FIG. 6. Calculated and experimental melting curves of $n$-octane. The simulation data were calculated by solving the Claussius-Clapeyron equation via the Gibbs-Duhem integration procedure. The integration was initiated using the coexistence point at $T=230 \mathrm{~K}$ obtained from the intersection of the chemical potential curves shown in Fig. 5. The gray zone marks the limits of uncertainty in the calculated melting curve. The procedure for the calculation of the uncertainty region is given in the text. The experimental data were taken from Ref. 52. The simulation melting curve data are also listed in Table IV. 
quantitative precision of the calculations is determined principally by the precision with which the solid and liquid phase free energies are calculated. However, the quantitative accuracy of the calculations, i.e., the degree to which the calculated and experimental data agree, is also expected to be highly sensitive to the details of the molecular potential employed in the calculations. Although the FW model was developed and optimized to reproduce various measured data for the solid and high-density liquid phases of alkanes, it is not clear that it would be adequate to calculate accurately the free energies required for the calculation of the solid-liquid phase boundary. The positive result of this study indicates both that a realistic all-atom model of this sort is sufficient, and that the free energy calculations themselves can be performed with sufficient precision, in order to predict accurately the melting curve for a $n$-alkane system.

\section{CONCLUSIONS AND OUTLOOK}

While the basic computational methods required to calculate the free energies of solids and liquids and, therefore, equilibrium solid-liquid phase behavior, have been available for many years, there has been little effort made to apply these methods to realistically modeled molecular systems. Work in this area has until now focused on simple, and mostly rigid, molecules. To our knowledge, the present study is the first to apply these methods to a truly realistic model for a system of large flexible molecules. The results look very promising: using a relatively simple all-atom alkane model we were able to obtain quantitatively accurate results for the melting curve of $n$-octane. While this system is still relatively simple compared to a polymer chain system, for example, it is considerably more complex than the diatomic or triatomic molecular systems studied to date. At present, a major limiting factor in these calculations is the large computational effort required to determine the melting curve for a realistic model oligomeric chain molecules such as $n$-octane. However, with the rapid increase in computing power, calculations that are barely feasible now should be standard in a few years time.

\section{ACKNOWLEDGMENTS}

This work is part of the research program of the "Stichting Fundamenteel Onderzoek der Materie" (FOM) and is supported by NWO ("Nederlandse Organisatie voor Wetenschappelijk Onderzoek"). J.P. acknowledges the financial support provided by the Computational Materials Science program of NWO, and by the Natural Sciences and Engineering Research Council of Canada. We would like to thank Andrew Rutenberg and Sander Pronk for a critical reading of the manuscript.

\section{APPENDIX: DEFINITION OF $U_{\mathrm{CH}}^{\mathrm{ID}}$ AND $Z_{\mathrm{CH}}^{\mathrm{ID}}$}

In this Appendix we describe in detail $U_{\text {ch }}^{\text {id }}$, the "ideal" component of the single-chain potential energy discussed in Sec. II B 2. We choose a $U_{\text {ch }}^{\text {id }}$ composed of bonded interaction terms of the following types:

- A bond-stretching term:

$$
U_{\mathrm{id}, b}=\frac{k_{\mathrm{id}, b}}{2}\left(r-l_{0}\right)^{2} .
$$

This is summed over $n-1 \mathrm{CC}$ bonds and $2 n+2 \mathrm{CH}$ bonds on each molecule, and is identical to the form which appears in the FW potential. We choose force constants and equilibrium bond lengths, to be equal in magnitude to those used in the FW potential: $k_{\mathrm{id}, b}=111200.0 \mathrm{~K} / \AA^{2}, l_{0}=1.530 \AA$ for $\mathrm{CC}$ bonds, and $k_{\mathrm{id}, b}=155800.0 \mathrm{~K} / \AA^{2}, \quad l_{0}=1.040 \AA$ for $\mathrm{CH}$ bonds.

- A CCC angle-bending term:

$$
U_{\mathrm{id}, \theta}^{\mathrm{CCC}}=\frac{k_{\mathrm{id}, \theta}^{\mathrm{CCC}}}{2}\left(\theta^{\mathrm{CCC}}-\theta_{0}^{\mathrm{CCC}}\right)^{2} .
$$

This term is summed over the $n-1$ CCC bond angles. Again, it is again identical in form, and with the same force constant and average CCC bond angles, as that employed in the FW potential: $k_{\mathrm{id}, \theta}^{\mathrm{CCC}}=62500.0 \mathrm{~K} / \mathrm{rad}^{2}$ and $\theta_{0}^{\mathrm{CCC}}=112^{\circ}$.

- A $\mathrm{CCH}$ angle bending term:

$$
U_{\mathrm{id}, \theta}^{\mathrm{CCH}}=\frac{k_{\mathrm{id}, \theta}^{\mathrm{CCH}}}{2}\left(\theta^{\mathrm{CCH}}-\theta_{0}^{\mathrm{CCH}}\right)^{2} .
$$

This term is summed only over $2 n+2 \mathrm{CCH}$ bond angles; that is, we include only one $\mathrm{CCH}$ bond angle per hydrogen atom. This choice effects a more straightforward analytical evaluation of the partition function. Further, the force constant magnitude is not set equal to that in the FW potential. To guarantee condition (2) for $U_{\mathrm{ch}}^{\text {id }}$ described in Sec. II B 2 we set $k_{i d, \theta}^{\mathrm{CCH}}=60000.0 \mathrm{~K} / \mathrm{rad}^{2}$. However, $\theta_{0}^{\mathrm{CCH}}=110.0^{\circ}$, as in the FW model.

- A CCH azimuthal angular term:

$$
U_{\mathrm{id}, \phi}=\frac{k_{\mathrm{id}, \phi}}{2}\left(\phi^{\mathrm{CCH}}-\phi_{0}^{\mathrm{CCH}}\right)^{2} \text {. }
$$

This term has the same constraining effect as an $\mathrm{HCH}$ angle bending potential, which is not included in $U_{\mathrm{ch}}^{\text {id }}$ (again, to simplify the evaluation of the partition function). $\phi^{\mathrm{CCH}}$ is the azimuthal angle of a $\mathrm{CH}$ bond relative to the carbon skeleton. This term is summed over $2 n$ azimuthal bond angles: $2(n$ -2) methylene $\phi^{\mathrm{CCH}}$ angles, and four of the six azimuthal angles associated with the terminal methyl groups. We take $k_{\mathrm{id}, \phi}=60000.0 \mathrm{~K} / \mathrm{rad}^{2}$ and $\phi_{0}^{\mathrm{CCH}}=120^{\circ}$.

- A methyl torsional potential term:

$$
U_{\mathrm{id}, \text { tor }}^{\text {meth }}=\frac{1}{2} E_{0}^{\text {meth }}\left(1-\cos 3 \phi^{\text {meth }}\right) .
$$

This torsional potential term is associated with the remaining one hydrogen per methyl group not included in the summation of the terms of Eq. (A4). The form of this potential is identical to that of the methyl rotational potential typically employed for constrained systems. We choose $E_{0}^{\text {meth }}$ $=1707.473 \mathrm{~K}$, as in Ref. 53 .

- A carbon skeleton torsional potential term:

$$
U_{\mathrm{id}, \mathrm{tor}}^{\mathrm{CCCC}}=\sum_{m=1}^{9} c_{m}^{\mathrm{CCCC}} \cos ^{(m)}\left(\phi^{\mathrm{CCCC}}\right) .
$$

This CCCC torsional potential is also chosen to be identical to that used in UA and constrained-AA alkane model potentials. The expansion coefficient values are taken from a fit to 
recent accurate spectroscopic data and ab initio calculations and are given in Ref. 54.

The partition function $Z_{\mathrm{id}}^{\mathrm{ch}}$ is given by

$$
Z_{\mathrm{ch}}^{\text {id }}=\left[\frac{V}{\Lambda_{\mathrm{C}}{ }^{3 n} \mathrm{C} \Lambda_{\mathrm{H}}{ }^{3 n_{\mathrm{H}}}}\right] Z_{\mathrm{ch}, \text { int }}^{\text {id }},
$$

where $\Lambda_{\mathrm{C}}$ and $\Lambda_{\mathrm{H}}$ are the de Broglie thermal wavelengths of each of the $n_{\mathrm{C}}$ carbon and $n_{\mathrm{H}}$ hydrogen atoms resulting from the integration of the momenta. The "internal" contribution to the single-chain ideal energy partition function $Z_{\mathrm{ch}, \text { int }}^{\text {id }}$, in the limit of stiff bonds, is given by

$$
\begin{aligned}
Z_{\mathrm{ch,int}}^{\mathrm{id}}= & 8 \pi^{2}\left[l_{\mathrm{CC}}^{2} \sqrt{\frac{2 \pi}{\beta k_{b}}}\left(1+\frac{1}{\beta k_{b} l_{\mathrm{CC}}^{2}}\right)\right]^{n-1}\left[l_{\mathrm{CH}}^{2} \sqrt{\frac{2 \pi}{\beta k_{b}}}\left(1+\frac{1}{\beta k_{b} l_{\mathrm{CH}}^{2}}\right)\right]^{2 n+2}\left[\int_{0}^{\pi} d \theta \sin \theta \exp \left[-\beta u_{\mathrm{id}, \theta}^{\mathrm{CCC}}\right]\right]^{n-2} \\
& \times\left[\int_{0}^{2 \pi} d \phi \exp \left[-\beta u_{\mathrm{id}, \phi}^{\mathrm{CCCC}}\right]\right]^{n-3}\left[\int_{0}^{\pi} d \theta \sin \theta \exp \left[-\beta u_{\mathrm{id}, \theta}^{\mathrm{CCH}}\right]\right]^{2 n+2}\left[\int_{0}^{2 \pi} d \phi \exp \left[-\beta u_{\mathrm{id}, \phi}^{m e t h}\right]\right]^{2} \\
& \times\left[\int_{0}^{2 \pi} d \phi \exp \left[-\beta u_{\mathrm{id}, \phi}\right]\right]^{2 n} .
\end{aligned}
$$

The five integrals in the above equation are evaluated numerically.

${ }^{1}$ B. Widom, J. Chem. Phys. 39, 2808 (1963).

${ }^{2}$ B. Widom, J. Chem. Phys. 86, 869 (1982).

${ }^{3}$ D. Frenkel and A. J. C. Ladd, J. Chem. Phys. 81, 3188 (1984).

${ }^{4}$ P. G. Bolhuis, D. Frenkel, S.-C. Mau, and D. A. Huse, Nature (London) 388, 235 (1997).

${ }^{5}$ D. Frenkel, B. M. Mulder, and J. P. McTague, Phys. Rev. Lett. 52, 287 (1984).

${ }^{6}$ D. Frenkel and B. M. Mulder, Mol. Phys. 55, 1171 (1985).

${ }^{7}$ J. A. C. Veerman and D. Frenkel, Phys. Rev. A 41, 3237 (1990).

${ }^{8}$ P. G. Bolhuis and D. Frenkel, J. Chem. Phys. 106, 666 (1997).

${ }^{9}$ S. J. Singer and R. Mumaugh, J. Chem. Phys. 93, 1278 (1990).

${ }^{10}$ C. Vega, E. P. A. Paras, and P. A. Monson, J. Chem. Phys. 96, 9060 (1992).

${ }^{11}$ C. Vega, E. P. A. Paras, and P. A. Monson, J. Chem. Phys. 97, 8543 (1992).

${ }^{12}$ E. J. Meijer, D. Frenkel, R. A. LeSar, and A. J. C. Ladd, J. Chem. Phys. 92, 7570 (1990)

${ }^{13}$ B. Kuchta, K. Rohleder, R. D. Etters, and J. Belak, J. Chem. Phys. 102, 3349 (1995).

${ }^{14}$ B. Kuchta, K. Rohleder, D. Swanson, and R. D. Etters, J. Chem. Phys. 106, 6771 (1997).

${ }^{15}$ B. Kuchta and R. D. Etters, Phys. Rev. B 47, 14691 (1993).

${ }^{16}$ B. Kuchta and R. D. Etters, Phys. Rev. B 45, 5072 (1992).

${ }^{17}$ L. A. Báez and P. Clancy, Mol. Phys. 86, 385 (1995).

${ }^{18}$ J. I. Siepmann, S. Karaborni, and B. Smit, Nature (London) 365, 330 (1993).

${ }^{19}$ B. Smit, S. Karaborni, and J. I. Siepmann, J. Chem. Phys. 102, 2126 (1995).

${ }^{20}$ M. G. Martin and J. I. Siepmann, J. Phys. Chem. B 102, 2569 (1998).

${ }^{21}$ B. Chen, M. G. Martin, and J. I. Siepmann, J. Phys. Chem. B 102, 2578 (1998).

${ }^{22}$ G. C. A. M. Mooij, D. Frenkel, and B. Smit, J. Phys.: Condens. Matter 4, L255 (1992).

${ }^{23}$ M. Laso, J. J. de Pablo, and U. W. Suter, J. Chem. Phys. 97, 2817 (1992).

${ }^{24}$ A. Z. Panagiotopoulos, Mol. Phys. 61, 813 (1987).

${ }^{25}$ A. Z. Panagiotopoulos, N. Quirke, M. Stapleton, and D. J. Tildesley, Mol. Phys. 63, 527 (1988).

${ }^{26}$ B. Smit, P. de Smedt, and D. Frenkel, Mol. Phys. 68, 931 (1989).
${ }^{27}$ J. I. Siepmann, Mol. Phys. 70, 1145 (1990).

${ }^{28}$ J. I. Siepmann and D. Frenkel, Mol. Phys. 75, 59 (1992).

${ }^{29}$ D. Frenkel, G. C. A. M. Mooij, and B. Smit, J. Phys.: Condens. Matter 4, 3053 (1991)

${ }^{30}$ A. P. Malanoski and P. A. Monson, J. Chem. Phys. 107, 6899 (1997)

${ }^{31}$ J. M. Polson and D. Frenkel, J. Chem. Phys. 109, 318 (1998).

${ }^{32}$ A. P. Malanoski and P. A. Monson, J. Chem. Phys. 110, 664 (1999).

${ }^{33}$ J. P. Ryckaert, Mol. Phys. 55, 549 (1985).

${ }^{34}$ J. P. Ryckaert, I. R. McDonald, and M. L. Klein, Mol. Phys. 67, 957 (1989).

${ }^{35}$ S. Toxvaerd, J. Chem. Phys. 93, 4290 (1990).

${ }^{36}$ S. Toxvaerd, J. Chem. Phys. 107, 5197 (1997).

${ }^{37}$ D. E. Williams, J. Chem. Phys. 47, 4680 (1967).

${ }^{38}$ J. C. Smith and M. Karplus, J. Am. Chem. Soc. 114, 801 (1992).

${ }^{39}$ D. J. Tobias, K. Tu, and M. L. Klein, J. Chim. Phys. Phys.-Chim. Biol. 94, 1482 (1997).

${ }^{40}$ M. Tuckerman, G. J. Martyna, and B. J. Berne, J. Chem. Phys. 93, 1287 (1990).

${ }^{41}$ M. Tuckerman, B. J. Berne, and G. J. Martyna, J. Chem. Phys. 97, 1990 (1992).

${ }^{42}$ G. J. Martyna, M. E. Tuckerman, D. J. Tobias, and M. L. Klein, Mol. Phys. 87, 1117 (1996).

${ }^{43}$ M. Watanabe and M. Karplus, J. Chem. Phys. 99, 8063 (1993).

${ }^{44}$ J. M. Polson, E. Trizac, S. Pronk and D. Frenkel (unpublished).

${ }^{45}$ D. A. Kofke, Mol. Phys. 78, 1331 (1993).

${ }^{46}$ D. A. Kofke, J. Chem. Phys. 98, 4149 (1993).

${ }^{47}$ A. Habenschuss and A. H. Narten, J. Chem. Phys. 91, 4299 (1989).

${ }^{48}$ H. Mathisen, N. Norman, and B. F. Pedersen, Acta Chem. Scand. 21, 9 (1967).

${ }^{49}$ G. J. Martyna, D. J. Tobias, and M. L. Klein, J. Chem. Phys. 10, 4177 (1994).

${ }^{50}$ J. P. Ryckaert, G. Ciccotti, and H. J. C. Berendsen, J. Comput. Phys. 23, 327 (1977).

${ }^{51}$ H. C. Andersen, J. Comput. Phys. 52, 24 (1983).

${ }^{52}$ A. Würflinger, Ber. Bunsenges. Phys. Chem. 79, 1195 (1975).

${ }^{53}$ R. A. Scott and H. A. Scheraga, J. Chem. Phys. 44, 3054 (1966).

${ }^{54}$ G. D. Smith and R. L. Jaffe, J. Phys. Chem. 100, 18718 (1996).

${ }^{55}$ N. Norman and H. Mathisen, Acta Chem. Scand. 15, 1755 (1961). 